\section{Pulsatile ocular \\ blood flow of \\ choroidal \\ neovascularization \\ in asymmetric age- \\ related macular \\ degeneration after \\ transpupillary \\ thermotherapy}

S-J Chen' ${ }^{1}$, C-Y Cheng ${ }^{1,2}$, A-F Lee', F-L Lee'1,

W-M Hsu' and J-H Liu'
'Department of Ophthalmology Taipei Veterans General Hospital National Yang-Ming University School of Medicine, Taipei, Taiwan

${ }^{2}$ Institute of Clinical Medicine National YangMing University

Taipei, Taiwan

Correspondence: S-J Chen Department of Ophthalmology Taipei Veterans General Hospital 201, Section 2 Shih-Pai Road Taipei 112, Taiwan Tel: + 886228757325 Fax: + 886228720959

E-mail: sjchen@

vghtpe.gov.tw

Received: 3 May 2003 Accepted: 24 July 2003

The authors have no proprietary interest in any aspect of this study.

Presented in part at the Annual Meeting of the Association for Research in Vision and Ophthalmology, May 2002, Fort Lauderdale, FL, USA 
However, it remains unanswered whether increased POBF leads to the development of CNV or vice versa. Given the higher POBF associated CNV and lower POBF associated with scarring, it would be interesting to evaluate how the POBF of eyes with CNV changes when the CNV shrinks to a disciform scar or progresses to a more destructive lesion. This information would help us better understand the haemodynamic role of the choroid in exudative AMD.

Current treatments for $\mathrm{CNV}$ with proven benefits include laser photocoagulation and photodynamic therapy. These therapies focus on the ablation of the CNV to minimize the destruction of the retina overlying the CNV. The favourable end result is limited scar tissue with no leakage apparent during fluorescein angiography and less destruction of the outer retina. Transpupillary thermotherapy (TTT), a technique currently under investigation, may also occlude the CNV without damage to other cells because less thermal energy is delivered. ${ }^{3}$ The normogram of treatment parameters for TTT requires further study using multicentre clinical trials. However, we have begun to use this therapy for most of our AMD patients with subfoveal classic or occult CNV.

Recent study using colour Doppler imaging showed decreased retinal blood flow $24 \mathrm{~h}$ after TTT and decreased choroidal perfusion 1 month after therapy. ${ }^{4}$ This study was designed to evaluate whether the POBF of eyes with CNV changes after TTT, relative to their pre-TTT treatment status and to contralateral eyes with drusen or scarring that have not been treated with TTT. It should be noted that this study was not designed to assess the efficacy of TTT.

\section{Materials and methods}

\section{Patients and definition}

The study patients and disease definition meet the criteria described in the previous study. ${ }^{2}$ In short, drusen are defined as in categories II and III of the Age-Related Eye Disease Study ${ }^{5}$ and CNV was distinguished as classic or occult $\mathrm{CNV}$ by evidence of leakage on fluorescein angiography. The fibrous components of CNV should be less than $25 \%$ of the total lesion. Scarring was classified as white fibrous tissue with blocked fluorescence or staining on angiography, and exceeding $75 \%$ of the total lesion.

Exclusion criteria for study subjects were a history of glaucoma, anisometropia, high-level myopia, a history of laser treatment for $\mathrm{CNV}$, or vitreoretinal surgery except for uneventful cataract surgery more than 1 year previously. Patients with arrhythmia that precluded stable recording of ocular pulse waves were also excluded. Eligible subjects underwent assessments of refraction, best-corrected visual acuity with a Snellen chart, slit-lamp biomicroscopy, axial length, fundus photography, and fluorescein angiography when enrolled in the study. Patients with asymmetric exudative AMD who had CNV in one eye were treated with TTT and followed monthly for 6 months or until a second TTT or other treatment was performed. Subjective visual performance, including best-corrected visual acuity, was recorded. Fundus biomicroscopy and fundus photography were used to evaluate the lesion on each visit. Fluorescein angiography was repeated on the surgeon's recommendation.

Informed consent was obtained from each patient who enrolled in the study, and the study was conducted according to the tenets of the Declaration of Helsinki of the World Medical Association regarding scientific research on human subjects.

\section{POBF measurement}

An ocular blood flow tonometer (Ocular Blood Flow Ltd.,. Wiltshire, UK) was used in this study to measure POBF. POBF and pulse amplitude (PA) were measured in all patients on each visit by an experienced examiner who was masked to the diagnosis and treatment.

Blood pressure was measured by sphygmomanometry with the patient in a sitting position. The mean blood pressure (MBP) was calculated as diastolic blood pressure plus $1 / 3$ of the systolic minus the diastolic blood pressure. The ocular perfusion pressure (OPP) was calculated as 2/3 MBP minus the intraocular pressure (IOP). ${ }^{6}$

\section{Transpupillary thermotherapy}

TTT was delivered through a slit lamp using an infrared laser at $810 \mathrm{~nm}$ with adjustable beam widths of 1.2,2.0, or $3 \mathrm{~mm}$ (Iris Medical Instruments, Mountain View, CA, USA) as described by Reichel et al. ${ }^{3}$ with some modification. The power was reduced to $60 \%$ of the typical treatment setting. In general, for a 3-mm spot size, the initial power level was between 450 and $600 \mathrm{~mW}$, and was adjusted depending on the type of $\mathrm{CNV}$, the amount of subretinal fluid (as estimated by biomicroscopy), the presence of pigment epithelial detachment, blood, or fibrosis. Treatment duration was $1 \mathrm{~min}$ and retinal colour was monitored intermittently so that no visible change or a barely detectable light-grey appearance to the lesion was present at the end of the treatment. If any retinal whitening was observed or the patient felt discomfort during the treatment, the power of the laser was decreased by $100 \mathrm{~mW}$ and the treatment was reinitiated. 
Retreatment with TTT was recommended when fundus biomicroscopic examination showed persistent subretinal fluid or exudation after 2-3 months of the initial therapy.

\section{Statistical analysis}

The paired Student's $t$-test was used to assess the difference in POBF and PA in CNV eyes before and after TTT, and to compare the differences in POBF and PA between contralateral eyes in the same subject at each visit. Multivariate regression analysis with a generalized estimating equation ${ }^{7}$ was performed to adjust for the effects of IOP, OPP, and pulse rate on POBF and PA. To minimize the variation in POBF measurements and to avoid the systemic factors that may affect interindividual variation, we used the contralateral eyes without TTT as controls in this study. That is, the difference in POBF between the two eyes of patients with asymmetric AMD was calculated (POBF of the CNV eye minus POBF of the contralateral eye), and then the difference in POBF was compared before and after TTT.

\section{Results}

In total, 26 patients with asymmetric exudative AMD were enrolled in this prospective case series. Of these, 20 subjects had a contralateral eye with drusen and six had scarring in the contralateral eye. The average age was $75.4 \pm 4.9$ years, and there were 24 male and two female subjects. The mean refractive difference between the two eyes was $0.1 \pm 1.8$ diopters. In all, 15 eyes had classic $\mathrm{CNV}$, eight eyes occult $\mathrm{CNV}$, and three eyes mixed types of CNV. The average size of the $\mathrm{CNV}$ was 4.8 disc areas (range: $1-9$ disc areas). During the follow-up period, five patients were retreated with TTT at 2-3 months after the initial therapy, and eight patients were lost to follow-up at 6 months.

Before TTT, the PA and POBF of eyes with CNV were greater than the values for contralateral eyes with drusen or scarring ( $P=0.046$ and $P<0.001$, respectively), as shown in Table 1. After adjustment for IOP, OPP, and PR, the POBF was still significantly $(P<0.001)$ different, although the difference in PA was no longer significant $(P=0.098)$. After TTT laser treatment, no significant difference was observed between pairs of eyes on each visit. When compared with pretreatment levels, the POBF of eyes with CNV was significantly decreased at 1 month $(P=0.002)$, reached a minimum at 2 months $(P=0.011)$, and rebounded after 3 months $(P=0.441)$.

The difference in POBF between the two eyes of AMD patients began to decrease 1 month after TTT, and reached the nadir at 2 months after TTT (Figure 1). The mean value for POBF in CNV eyes minus the value for the contralateral eyes was $245.9 \mu \mathrm{l} / \mathrm{min}$ before TTT, and

Table 1 Mean values (SD) of IOP, OPP, PA, and POBF before and after TTT

\begin{tabular}{|c|c|c|c|c|c|}
\hline & $\begin{array}{l}\text { Pretreatment } \\
\quad(n=26)\end{array}$ & $\begin{array}{l}1 \text { month after } \\
\text { TTT }(n=20)\end{array}$ & $\begin{array}{c}2 \text { months after } \\
\text { TTT }(n=16)\end{array}$ & $\begin{array}{c}3 \text { months after } \\
\text { TTT }(n=10)\end{array}$ & $\begin{array}{c}6 \text { months after } \\
\text { TTT }(n=13)\end{array}$ \\
\hline \multicolumn{6}{|l|}{ IOP $(m m H g)$} \\
\hline $\mathrm{CNV}$ & 13.5 (3.5) & $15.3(5.7)$ & $14.7(2.8)$ & $15.6(3.7)$ & $14.6(4.8)$ \\
\hline Contralateral & $15.6(3.9)$ & $15.9(4.8)$ & $13.7(3.2)$ & $16.8(3.7)$ & $14.2(3.4)$ \\
\hline \multicolumn{6}{|l|}{ eye } \\
\hline$P$-value ${ }^{\mathrm{a}}$ & 0.001 & 0.489 & 0.368 & 0.328 & 0.777 \\
\hline \multicolumn{6}{|l|}{ OPP ( $(m m H g)$} \\
\hline $\mathrm{CNV}$ & $51.6(9.1)$ & $49.8(11.2)$ & $49.0(8.6)$ & $45.6(9.4)$ & 49.9 (10.5) \\
\hline Contralateral & $49.5(8.3)$ & $49.2(9.4)$ & $50.0(7.9)$ & $44.5(9.0)$ & $50.3(9.6)$ \\
\hline \multicolumn{6}{|l|}{ eye } \\
\hline$P$-value ${ }^{\mathrm{a}}$ & 0.001 & 0.488 & 0.368 & 0.328 & 0.777 \\
\hline \multicolumn{6}{|l|}{$P A(m m H g)$} \\
\hline $\mathrm{CNV}$ & $3.5(1.7)$ & $3.2(0.9)$ & $2.8(1.3)^{\mathrm{b}}$ & $3.2(1.7)$ & $2.6(1.5)$ \\
\hline Contralateral & $3.1(1.2)$ & $3.4(1.1)$ & $2.6(1.0)$ & $3.4(1.3)$ & $2.5(1.2)$ \\
\hline \multicolumn{6}{|l|}{ eye } \\
\hline$P$-value ${ }^{\mathrm{a}}$ & 0.046 & 0.2 & 0.167 & 0.302 & 0.602 \\
\hline \multicolumn{6}{|l|}{ POBF $(\mu \mathrm{l} / \mathrm{min})$} \\
\hline $\mathrm{CNV}$ & 1178.5 (317.2) & $1014.5(272.8)^{\mathrm{b}}$ & $945.3(398.3)^{b}$ & $1096.0(348.4)$ & $1010.6(396.3)$ \\
\hline Contralateral & $933.6(280.7)$ & 1036.7 (239.5) & 1083.2 & $1086.3(329.6)$ & $917.3(344.3)$ \\
\hline \multicolumn{6}{|l|}{ eye } \\
\hline$P$-value ${ }^{\text {a }}$ & $<0.001$ & 0.586 & 0.313 & 0.891 & 0.185 \\
\hline
\end{tabular}

$\overline{\mathrm{SD}}=$ standard deviation; $\mathrm{TTT}=$ transpupillary thermotherapy; $\mathrm{OPP}=$ ocular perfusion pressure; $\mathrm{PA}=\mathrm{pulse}$ amplitude; $\mathrm{POBF}=$ pulsatile ocular blood flow.

a'Comparison between eyes with CNV and contralateral eyes using the paired Student's $t$-test.

bSignificantly different $(P<0.05)$ before and after TTT treatment by paired Student's $t$-test. 


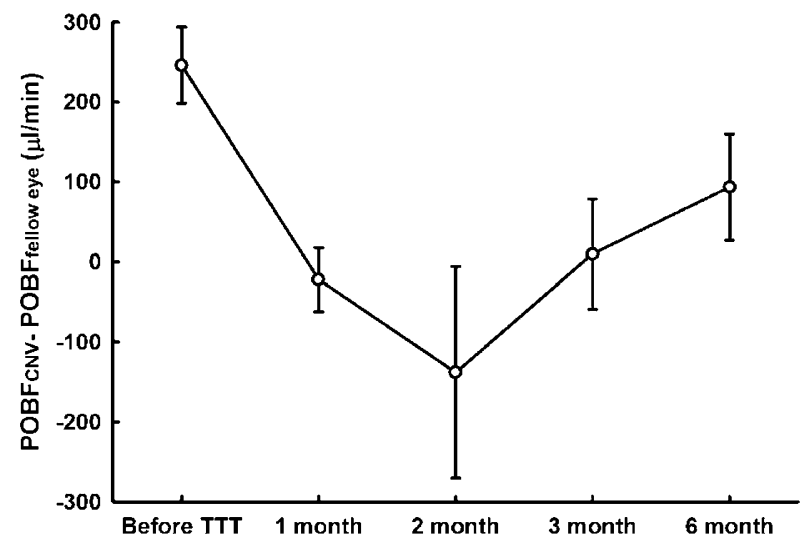

Figure 1 Difference in pulsatile ocular blood flow (mean \pm SE) between the two eyes of subjects before and after TTT at 1, 2, 3, and 6 months.

decreased to $-22.2 \mu \mathrm{l} / \mathrm{min}$ at 1 month $(P<0.001)$, $-137.9 \mu \mathrm{l} / \mathrm{min}$ at 2 months $(P=0.029), 9.7 \mu \mathrm{l} / \mathrm{min}$ at 3 months $(P=0.005)$, and $93.3 \mu \mathrm{l} / \mathrm{min}$ at 6 months $(P=0.078)$ after TTT laser treatment.

\section{Discussion}

Our results show that TTT transiently reduced the POBF in treated eyes compared with their pretreatment status and with the contralateral eyes of individuals with asymmetric exudative AMD. The effects began to appear at 1 month and were most prominent at 2 months after treatment.

In the previous study, ${ }^{2}$ we speculated that the pulsatile components of the choroidal blood flow were dynamically influenced during the development of exudative AMD. This study has shown that POBF and PA in eyes with CNV were still lower than their pretreatment values at 6 months after TTT, even though the recovery had rebounded. In contrast, we found that POBF and PA were quite stable in patients with macular scarring during the 6-month follow-up period in a small series study (unpublished data). A decreased serial POBF might indicate that the choroidal blood flow is diminished when CNV evolves into a scar.

The mechanism underlying decreased POBF after TTT is unknown. Three possibilities might explain this finding. One is that the scleral rigidity decreases after laser treatment, so that the measured pulsation is reduced. How scleral rigidity changes after laser treatment of the macula is unclear. However, as fibrosis increases and blood vessels decreases during the formation of scar tissue, scleral rigidity should increase because there is less buffer effect from the choroidal vascular channels. ${ }^{8}$ Another explanation is that the pulsatile components of the choroidal blood flow shift to nonpulsatile components after laser treatment, as observed in normal volunteers after the Valsava manoeuver. ${ }^{9}$ The third possible reason is that the total choroidal blood flow decreases after TTT. With colour Doppler imaging, Ciulla and Harris demonstrated an increased resistance index and a decreased end-diastolic velocity in the short posterior ciliary arteries 1 month after TTT. ${ }^{4}$ Although only the changes in the nasal branch were statistically significant, the decrease in peak systolic velocity and end-diastolic velocity in both the nasal and temporal branches (as shown in the table of their report) might also imply a reduced bulk flow in the retrobulbar vessels. The clinical data were not reported in their study.

In our series, the reduced POBF reached a minimum at 2 months. However, in studies using fluorescein and indocyanine green angiography, a decrease in leakage was observed from 1 day to 1 month after TTT. ${ }^{10}$ Hence, a change in the microenvironment of the CNV and a resolution of the fluid after treatment might precede the change in choroidal blood flow. As the blood flow change lasted no longer than 3 months, it will be appropriate to consider retreatment with TTT at 2-3 months after the initial therapy if there is no evidence of clinical improvement.

It will be interesting to see if maintaining the POBF of $\mathrm{CNV}$ at a lower level decreases the progression of $\mathrm{CNV}$, and whether other pharmacological or surgical treatments also facilitate a decrease in pulsation in eyes with CNV. POBF might also be helpful in evaluating treatment effects. These questions will be addressed by further studies.

It is less clear whether increased POBF leads to CNV formation. Five patients in this study underwent secondary TTT for persistent or increased subretinal fluid, or vitrectomy for massive submacular haemorrhage. The POBF and PA in the eyes with $\mathrm{CNV}$ of four patients before retreatment increased relative to their previous measurements. However, the patient numbers were too small, and a long-term follow-up study of patients with bilateral or unilateral drusen will be required to evaluate whether increased POBF predisposes patients to the development of CNV.

POBF measurements displayed high interindividual variation and a moderate reliability of $70 \%$ when tested one day apart. ${ }^{11}$ To avoid systemic factors that may affect interindividual variation, it was better to compare POBF between contralateral eyes with different disease states in the same individual, instead of comparing groups of patients with and without exudative AMD. ${ }^{1,2}$ In this study, we assumed that the POBF of the contralateral eye with drusen or scarring was stable during the follow-up period, so that after TTT, the difference between the eye with $\mathrm{CNV}$ and the contralateral eye with drusen or scarring could be assessed. Throughout the study period, 
up until the preparation of this manuscript, no CNV developed in any contralateral eye.

In conclusion, this study shows that TTT decreases the pulsatile choroidal blood flow in eyes of asymmetric AMD patients with $\mathrm{CNV}$ and that the effects last for 2 months. Although further study is warranted to elucidate the vascular pathogenesis of exudative AMD, POBF may be used as a modality to monitor the therapeutic effects of $\mathrm{CNV}$ in asymmetric exudative AMD.

\section{References}

1 Mori F, Konno S, Hikichi T, Yamaguchi Y, Ishiko S, Yoshida A. Pulsatile ocular blood flow study: decreases in exudative age-related macular degeneration. Br J Ophthalmol 2001; 85: 531-533.

2 Chen SJ, Cheng CY, Lee AF, Chou JC, Hsu WM, Liu JH. Pulsatile ocular blood flow in asymmetric exudative agerelated macular degeneration. Br J Ophthalmol 2001; 85: 1411-1415.

3 Reichel E, Berrocal AM, Ip M, Kroll AJ, Desai V, Duker JS et al. Transpupillary thermotherapy of occult subfoveal choroidal neovascularization in patients with age-related macular degeneration. Ophthalmology 1999; 106: 1908-1914.
4 Ciulla TA, Harris A, Kagemann L, Danis RP, Maturi R, McNulty L et al. Transpupillary thermotherapy for subfoveal occult choroidal neovascularization: effect on ocular perfusion. Invest Ophthalmol Vis Sci 2001; 42: 33373340.

5 The Age-Related Eye Disease Study Research Group. The Age-Related Eye Disease Study (AREDS): design implications. AREDS report no. 1. Control Clin Trials 1999; 20: $573-600$.

6 Williamson TH, Harris A. Ocular blood flow measurement. Br J Ophthalmol 1994; 78: 939-945.

7 Liang KY, Zeger SL. Longitudinal data analysis using generalized linear models. Biometrics 1986; 73: 13-22.

8 Kiel JW. The effect of arterial pressure on the ocular pressure-volume relationship in the rabbit. Exp Eye Res 1995; 60: 267-278.

9 Schmetterer L, Dallinger S, Findl O, Strenn K, Graselli U, Eichler HG et al. Noninvasive investigations of the normal ocular circulation in humans. Invest Ophthalmol Vis Sci 1998; 39: 1210-1220.

10 Lanzetta P, Michieletto P, Pirracchio A, Bandello F. Early vascular changes induced by transpupillary thermotherapy of choroidal neovascularization. Ophthalmology 2002; 109: 1098-1104.

11 Yang YC, Hulbert MF, Batterbury M, Clearkin LG. Pulsatile ocular blood flow measurements in healthy eyes: reproducibility and reference values. J Glaucoma 1997; 6: 175-179. 